Pacific Journal of Mathematics

SEMIMODULARITY IN THE COMPLETION OF A POSE 


\section{SEMIMODULARITY IN THE COMPLETION OF A POSET}

\section{Barbara Jeffcott and William T. Spears}

M. D. MacLaren examined semimodularity in the completion by cuts of a lattice $L$, and showed that if $L$ is semimodular, atomic, and orthocomplemented then $\bar{L}$ is semimodular [Pacific J. Math. 14 (1964)]. We study here semimodularity in an orthomodular poset $P$ and its completion by cuts $\bar{P}$. In particular, we show that if $P$ is semimodular and orthomodular and contains no infinite chains, then $\bar{P}$ is semimoduląr if and only if $\bar{P}$ is isomorphic to $P$. Hence, contrary to the result of MacLaren for lattices, semimodularity is never preserved in the completion by cuts of an orthomodular poset with no infinite chains which is not a lattice. More generally, we show that if $P$ is orthomodular, atomic, and orthocomplete, then the covering condition in $P$ is carried over to $\bar{P}$ if and only if $P$ is isomorphic to $\bar{P}$. As a result, MacLaren's theorem cannot be generalized to posets.

We obtain these results by constructing a new, more convenient way of viewing the completion by cuts of a poset. In $\$ 4$ we use this characterization of the completion by cuts to provide examples which show that if either the condition that $P$ is atomic, or the condition that $P$ is orthocomplete is removed, then the theorem fails; that is $P$ may fail to be a lattice but both $P$ and $\bar{P}$ may satisfy the covering condition.

Let $P$ be any partially ordered set. For each subset $X \subseteq P$ define $X^{u}$ to be $\{t \in P: t \geqq x$ for all $x \in X\}$ and define $X^{1}=\{t \in P: t \leqq x$ for all $x \in X\}$. Write $X^{u 1}$ for $\left(X^{u}\right)^{1}$. Then the completion by cuts of $P$ is the complete lattice $\bar{P}=\left\{X^{u 1}: X \subseteq P, X \neq \emptyset\right\}$, ordered by set inclusion [5].

It is straightforward to show that if $P \rightarrow P: x \rightarrow x^{\prime}$ is an orthocomplement on $P$, then ${ }^{*}: \bar{P} \rightarrow \bar{P}: X^{u 1} \rightarrow\left\{x^{\prime}: x \in X\right\}^{1}$ is an orthocomplement on $\bar{P}$ [c.f. 4, MacLaren]. P. D. Finch extended this result by providing necessary and sufficient conditions for the conpletion by cuts of an orthocomplemented poset to be orthomodular [2, Proposition 3.2]. Our Theorem 3.6 also shows the relationship between orthomodularity and the covering condition in $\bar{P}$.

2. Definitions. If $a$ and $b$ are elements of a partially ordered set, write $a<b$ to mean that $b$ covers $a$. A lattice $L$ with zero is said to satisfy the covering condition if whenever $a$ is an atom of $L$ and and $b \in L$ with $a \wedge b=0$, then $b<a \vee b$. As a natural generaliza- 
tion of this definition, a poset $P$ with zero will be said to satisfy the covering condition if whenever $a$ is an atom of $P$ and $b \in P$ with $a \neq b$, then some upper bound of $a$ covers $b$. A lattice $L$ with zero having no infinite chains is called upper semimodular or a Birkhoff lattice if it satisfies the property that for all elements $a$ and $b$, if $a \wedge b<a$ and $a \wedge b<b$, then $a<a \vee b$ and $b<a \vee b$. Birkhoff extended this definition to call a poset $P$ with zero having no infinite chains upper semimodular if it satisfies the condition that whenever $a, b, c \in P$ with $c<a$ and $c<b$ then there exists $d \in P$ with $a<d$ and $b<d$.

If $L$ is a lattice having no infinite chains, then upper semimodularity is equivalent to the covering condition. Furthermore, a lattice satisfying these properties is always $M$-symmetric. Conversely, every $M$-symmetric lattice satisfies the covering condition. Hence a lattice $L$ (perhaps having infinite chains) is defined to be upper semimodular if and only if it is $M$-symmetric. (All the above definitions and theorems may be found in Birkhoff, [1]).

Haskins and Gudder have shown that a similar relationship between the definitions holds in posets with no infinite chains as does in lattices [3, Theorem 3.8]. In particular, they show that if $P$ is a connected poset with zero having no infinite chains, then $P$ is upper semimodular if and only if whenever $a, b \in P$ with $a \not b$, and $a$ covers a lower bound of $b$, then some upper bound of $a$ covers $b$. It is straightforward to show that in any orthomodular poset, their second condition is equivalent to the covering condition. Since we will be dealing only with orthomodular posets $P$, we have, as in lattices, that if $P$ has no infinite chains, then $P$ is upper semimodular if and only if it satisfies the covering condition. On the other hand, since $M$-symmetry is not defined in a poset, we content ourselves with studying the covering condition in posets having infinite chains.

Lower semimodularity and the dual covering condition are defined dual to upper semimodularity and the covering condition in both posets and lattices. $P$ or $L$ is called semimodular if it is both upper semimodular and lower semimodular. Note that if $P$ or $L$ is orthocomplemented, then it is semimodular if and only if it is either upper semimodular or lower semimodular, and it satisfies the covering condition, if and only if it satisfies the dual covering condition, if and only if it satisfies both of them.

3. The completion by cuts. We begin by characterizing the completion by cuts of an arbitrary poset. If $L$ is a complete lattice and $P$ is any subset of $L$ containing 0 and 1 , then for each subset $X \subseteq L$ we will write $X^{u}$ for $\{p \in P: p \geqq x$ for all $x \in X\}$ and $X^{1}=$ $\{p \in P: p \leqq x$ for all $x \in X\}$. Write $X^{u 1}$ for $\left(X^{u}\right)^{1}$, etc. Note that no confusion arises here by using the same notation as in the completion by 
cuts of a poset, for if $P$ is given the induced ordering from $L$ and $X \subseteq P$, then $X^{u 1}$ is the same set under either definition.

THEOREM 3.1. Let $L$ be a complete lattice, and let $P$ be a subset of $L$ containing 0 and 1. Provide $P$ with the induced ordering. Define $\phi: \bar{P} \rightarrow L$ by $\phi\left(X^{u 1}\right)=\vee\left(X^{u 1}\right)$. Then $\phi$ is an isomorphism if and only if for all $a \in L, a=\wedge a^{u}=\vee a^{\prime}$.

Proof. Assume first that $\phi$ is an isomorphism. Let $a \in L$. Then there exists $X \subseteq P$ with $\phi\left(X^{u 1}\right)=a=\vee X^{u 1}$. Then $X^{u 1} \subseteq a^{1}$, so $a=$ $\vee X^{u 1} \leqq \vee a^{1} \leqq a$. Hence $a=\vee a^{1}$. Now let $m=\wedge a^{u} \in L$. There exists subsets $X, Y \subseteq P$ satisfying the conditions $a=\phi\left(X^{u 1}\right)$ and $m=$ $\phi\left(Y^{u 1}\right)$. But $X^{u 1}=\vee_{x \in X} X^{u 1}$, as computed in $\bar{P}$, and $\phi$ is an isomorphism, so $a=\phi\left(X^{u 1}\right)=\phi\left(\vee_{x \in X} x^{u 1}\right)=\vee_{x \in X} \phi\left(x^{u 1}\right)$. But $X \subseteq P$, so $x \in X$ implies $x=v x^{1}=\vee x^{u 1}=\phi\left(x^{u 1}\right)$. Hence $a=\vee X$. Similarly, $m=$ $\vee Y$. Hence $a^{u}=X^{u}$ and $m^{u}=Y^{u}$. But $a^{u}=m^{u}$ since $m=\wedge a^{u}$, so $X^{u 1}=Y^{u 1}$ and it foltows that $a=m$. Thus $a=\wedge a^{u}$.

Conversely, assume that for all $a \in L, a=\wedge a^{u}=\vee a^{1}$. First note that if $a \in L$ then $a^{1}=a^{u 1}$. To see this, let $x \in a^{u 1}$. Then since $x \leqq t$ for all $t \in a^{u}$, it follows that $x \leqq \wedge a^{u}=a$, so $x \in a^{1}$. Clearly $a^{1} \subseteq a^{u 1}$, so we have equality. We next note that if $\varnothing \neq X \subseteq L$, then $\vee X^{u 1}=$ $\vee(\vee X)^{1}=\vee X$. Too see this, observe first that since $X^{u}=(\vee X)^{u}$, it follows that $X^{u 1}=(\vee X)^{u 1}$. Since $\vee X \in L$, by the first argument, $X^{u 1}=$ $(\vee X)^{u 1}=(\vee X)^{1}$, and hence $\vee X^{u 1}=\vee\left[(\vee X)^{1}\right]$. But by hypothesis, $\vee X=$ $\vee\left[(\vee X)^{\prime}\right]$.

The map $\phi$ is clearly well defined and isotone. To show it is a surjection, fix $b \in L$. Then $\emptyset \neq b^{1} \subseteq L$, so by the above argument, $\vee\left(b^{1 u^{1}}\right)=v b^{1}$. Hence by hypothesis, $\phi\left[\left(b^{1}\right)^{u 1}\right]=b$, and $\phi$ is surjection. Now fix nonempty subsets $X, Y \subseteq P$ with $\vee X^{u 1} \leqq \vee Y^{u 1}$ in L. If $x \in X^{u 1}$ then $x \leqq \vee X^{u 1} \leqq \vee Y^{u 1}$, so it again follows from the arguments of the last paragraph that $x \leqq \vee Y$, and thus $x \in(\vee Y)^{1}=$ $Y^{u 1}$. Hence $X^{u 1} \leqq Y^{u 1}$ in $\bar{P}$. Thus $\phi$ and $\phi^{-1}$ are isotone and $\phi$ is a bijection; that is, $\phi$ is an isomorphism.

The above theorem makes it much easier to work with the completion by cuts. To simplify our work further, we make the following definition:

Definition 3.2. Let $P$ be a poset with 0 and 1. A completion for $P$ is a pair $(L, \sigma)$ where $L$ is a complete lattice and $\sigma: P \rightarrow L$ is an isotone map satisfying the following properties:

(1) $\sigma$ is an injection and $\sigma^{-1}$, restricted to $\sigma(P)$, is isotone.

(2) $\sigma$ preserves existing suprema and infima.

(3) For each $a \in L, a=\vee\{\sigma(p): p \in P$ and $\sigma(p) \leqq a\}$ and $a=$ $\wedge\{\sigma(p): p \in P$ and $\sigma(p) \geqq a\}$. 
Clearly if $P \subseteq L$, satisfies the conditions of the theorem, then $L$ together with the inclusion map is a completion for $P$. Similarly, if we define $\mu: P \rightarrow \bar{P}: p \rightarrow p^{1}$, then $(\bar{P}, \mu)$ is a completion for $P$. It follows immediately from the theorem that if $\left(L_{1}, \sigma_{1}\right)$ and $\left(L_{2}, \sigma_{2}\right)$ are both completions for $P$, then there exists a unique isomorphism $\theta: L_{1} \rightarrow L_{2}$, such that $\theta \sigma_{1}=\sigma_{2}$.

Now assume that ' $P \rightarrow P$ is an orthocomplement on $P$ and that $(L, \sigma)$ is a completion for $P$. Note that a unique orthocomplement $\#: L \rightarrow L$ can be defined which satisfies the condition that $\sigma(p)^{*}=$ $\sigma\left(p^{\prime}\right)$ for all $p \in P$. Furthermore, if $\left(L_{1}, \sigma_{1}\right)$ and $\left(L_{2}, \sigma_{2}\right)$ are completions for $P$, and *: $L_{1} \rightarrow L_{1}$ and \#: $L_{2} \rightarrow L_{2}$ are these induced orthocomplements, then the unique isomorphism $\theta: L_{1} \rightarrow L_{2}$ satisfying $\theta \sigma_{1}=\sigma_{2}$ is an or tho-isomorphism; that is, $\theta\left(a^{*}\right)=\theta(a)^{*}$ for all $a \in L_{1}$.

Clearly the easiest completion to work with is one of the form $(L, i)$, where $i$ is an inclusion. To summaize when we say that $L \supseteq P$ is a completion for $P$, we mean that $P$ is a subposet of $L$, that given any subset of $P$ its supremem or infimum (if it exists in $P$ ) is the same whether computed in $P$ or $L$, that for any $a \in L, a=\vee\{x \in P: x \leqq a\}=$ $\wedge\{x \in P: x \geqq a\}$, and that if $P$ is orthocomplemented then $L$ is orthocomplemented and the restriction of the orthocomplement of $L$ to $P$ is that of $P$. Then by the preceding remarks, any properties which hold for such a completion $L \supseteq P$, hold for the standard completion by cuts, $\bar{P}$, as defined earlier.

Lemma 3.3. Let $L \supseteq P$ be a completion for $P$. Assume further that $P$ is an orthomodular poset (in its own right) and that $L$ satisfies the covering condition. Then if $x, y \in P$ and $x<y$ in $P$, then $x<y$ in $L$.

Proof. Fix $x, y \in P$ with $x<y$ in $P$. Then clearly $y \wedge x^{\prime}$ is an atom of $P$. To show it is an atom of $L$, suppose there exists $b \in L$ with $b \neq 0$ and $b \leqq y \wedge x^{\prime}$. Then $b=\vee b^{1}=\vee\{p \in P: p \leqq b\}$. Since $b \neq 0$, there exists a nonzero $p \in P$ with $p \leqq b \leqq y \wedge x^{\prime}$. But $y \wedge x^{\prime}$ is an atom of $P$, so there exists exactly one such $p$, namely $y \wedge x^{\prime}$. Hence $b=$ $y \wedge x^{\prime}$. Hence $y \wedge x^{\prime}$ is an atom of $L$. By the covering condition in $L, y \wedge x^{\prime} \not \geq x$ implies that $\left(y \wedge x^{\prime}\right) \vee x>x$ in $L$. But $x, y \in P$ and $P$ orthomodular implies that $y=\left(y \wedge x^{\prime}\right) \vee x$, so $x<y$ in $L$.

Lemma 3.4. Same hypotheses as Lemma 3.3. Assume further that $P$ satisfies the covering condition. Then $a \vee y \in P$ for every atom $a$ in $P$ and for every $y \in P$.

Proof. Let $a$ be an atom of $P$ and $y \in P$. We may assume that $a \nsubseteq y$. Then there exists $x \in P$ with $a \leqq x$ and $y<x$. Since 
$x \in L, a \vee y \leqq x$. But $y \leqq a \wedge y \leqq x$ and by the last lemma $y<x$ in $L$, so $a \vee y=x \in P$.

Lemma 3.5. Let $L \supseteq P$ be a completion for $P$. Assume further that $P$ is orthomodular, orthocomplete and atomic, and that $a \vee y \in P$ for every atom $a \in P$ and for every $y \in P$. Then $P=L$.

Proof. For each $c \in L$ let $A_{c}=\{x \in P: x \leqq c, x$ is an atom $\}$. It is straightforward to show that $P$ is atomistic, and hence $p=\vee A_{p}$ for all $p \in P$. Now fix $b \in L$. Then $\cup_{y \in b^{1}} A_{y} \subseteq A_{b}$, so $b=\vee b^{1}=$ $\vee_{y \in b^{\prime}}\left(\vee A_{y}\right) \leqq \vee A_{b}$. But clearly $\vee A_{b} \leqq b$, so $b=\vee A_{b}$. Now let $C$ denote a maximal $\perp$ subset of $A_{b}$. Then $\vee C \in P$ and $\vee C \leqq b$. We claim that $b \in P$, and in fact that $b=\vee C$. Suppose that $b \notin P$. Then $\vee C \equiv b$. Since $b=\vee A_{b}$, there exists $x \in A_{b}$ with $x \notin \vee C$. By hypothesis, $x \vee(\vee C) \in P$. Now $P$ is orthomodular, and $\vee C \leq x \vee(\vee C)$, so $[x \vee(\vee C)] \wedge(\vee C)^{\prime} \neq 0$. Call this element $z$. Then $z \neq 0$ implies that $A_{z} \neq \varnothing$, so let $y \in A_{z}$. Then $y \leqq z \leqq(\vee C)^{\prime}$ implies that $y \perp c$ for all $c \in C$. So by the maximality of $C, y \notin A_{b}$. Hence $y \not b$, which is a contradiction.

We are now ready to prove our main theorem.

THEOREM 3.6. Let $P$ be an atomic, orthomodular, orthocomplete poset with the covering condition. Then the following five conditions are equivalent :

(1) $\bar{P}$ satisfies the covering condition.

(2) $P$ is a lattice.

(3) $P$ is a complete lattice.

(4) $P$ is orthoisomorphic to $\bar{P}$.

(5) $\bar{P}$ is orthomodular.

Furthermore, if $P$ has no infinite chains, then the above conditions and the conditions listed below are all equivalent.

(6) $\bar{P}$ is modular.

(7) $\bar{P}$ is M-symmetric.

The proof that (1) - (4) are equivalent follows immediately from Theorem 3.1, Lemma 3.3, Lemma 3.4 and the preceding remarks. Clearly (4) implies (5). Now let $L \supseteq P$ be a completion for $P$, and assume that $\bar{P}$, and hence $L$, is orthomodular. Suppose there exists $y \in L$ with $y \notin P$. Let $C$ be a maximal $\perp$ subset of atoms in $P$ under $y$. Then $\vee C \in P, \vee C \leqq y$ and $y \wedge(\vee C)^{\prime}=\vee\{p \in P \mid p \leqq$ $\left.y \wedge(\vee C)^{\prime}\right\} \neq 0$. So there exists an atom $p \in P$ with $p \leqq y \wedge(\vee C)^{\prime}$, which contradicts the maximality of $C$. The remainder of the proof follows from well known properties of lattices [c.f.1, Birkhoff p. 41]. 
4. Examples. We show here that the conditions of Theorem 3.6 may not be weakened. That is, we provide examples of nonatomic, orthomodular, orthocomplete posets with the covering condition for which the theorem fails, and examples of atomic, orthomodular posets with the covering condition which fail to be orthocomplete and for which the theorem fails.

EXAMPLE 4.1. Let $Q$ be any finite orthomodular poset which is not a lattice. Let $R$ be an infinite product of $Q$; that is, let $R=$ $\{f: N \rightarrow Q: f$ is a function $\}$, where $N$ denotes the natural numbers. Define an equivalence relation on $R$ by $f \equiv g$ if and only if $\{n \in N: f(n) \neq g(n)\}$ is finite. For $f \in R$, write $[f]$ for the equivalence class containing $f$, and let $P=\{[f]: f \in R\}=R / \equiv$. Define a partial order on $P$ by $[f] \leqq[g]$ if and only if $\{n \in N: f(n) \equiv g(n)\}$ is finite. Note that [0], where $0(n)=0$ for all $n \in N$ is effective as the zero of $P$. Furthermore, $f \equiv 0$ if and only if $\{n \in N: f(n) \neq 0\}$ is finite. Similarly, [1], defined by $1(n)=1$ for all $n \in N$, is effective as the one of $P$, and $g \equiv 1$ if and only of $\{n \in N: g(n) \neq 1\}$ is finite. Now define ' $^{\prime} P \rightarrow P$ by $[f]^{\prime}=[g]$, where $g(n)=f(n)^{\prime}$ for all $n \in N$, where $f(n)^{\prime}$ is the orthocomplement of $f(n)$ in $Q$. It is straightforward to show that ': $P \rightarrow P$ is well defined and that it is effective as an orthocomplement on $P$. Note that $[f] \vee[g]$ exists in $P$ if and only if $\{n \in N: f(n) \vee g(n)$ fails to exist in $Q\}$ is finite, and in this case $[f] \vee[g]=[h]$, for any $h \in R$ satisfying $h(n)=f(n) \vee g(n)$ for all $n \in N$ where $f(n) \vee g(n)$ exists in $Q$. A similar result holds for infimums. From the above comments it is straightforward to show that $P$ is an orthomodular poset.

We claim now that $P$ is orthocomplete. To see this, note first that since $Q$ is finite, every maximal orthogonal family of elements of $Q$ is finite. Assume that the largest family of pairwise orthogonal elements in $Q$ contains $k$ elements. Now suppose that $\left\{\left[h_{i}\right]: i=1,2, \ldots, k+1\right\}$ is a pairwise orthogonal family of elements in $P$. Now for each $i=1, \ldots, k+1,\left\{n \in N: h_{i}(n) \not h_{j}(n)\right\}$ is finite for each $j=1, \cdots, k+1$ with $j \neq i$. Hence

$$
\bigcup_{i=1}^{k+1} \bigcup_{\substack{i=1 \\ j \neq i}}^{k+1}\left\{n \in N: h_{1}(n) \not \subset h_{j}(n)\right\}
$$

is a finite set. Hence there exists $m \in N$ - in fact an infinite number of $m \in N$ - such that $m$ is not in this set; that is $\left\{h_{i}(m): i=1, \ldots, k+1\right\}$ is a pairwise orthogonal family of elements of $Q$. But the largest family of pairwise orthogonal elements in $Q$ contains $k$ elements, which is a contradiction. Hence there are at most a finite number, and in fact at 
most $k$ elements in any family of pairwise orthogonal elements of $P$. Hence $P$ is orthocomplete, vacuously.

Now suppose that $[f]$ is an atom of $P$. Then $\{n \in N: f(n) \neq 0\}$ is an infinite set, so there exist disjoint infinite sets $A$ and $B$ satisfying the condition that $A \cup B=\{n \in N: f(n) \neq 0\}$. Define $g \in R$ by $g(n)=0$ for $n \in B$, and $g(n)=f(n)$, otherwise. Then $[g] \neq[0],[g] \leqq[f]$, but $[f] \neq[g]$, which is a contradiction. Hence $P$ has no atoms.

We have constructed an example of a nonatomic orthocomplete, orthomodular poset $P$ for which both $P$ and $\bar{P}$ satisfy the covering condition. Furthermore, since $Q$ is not a lattice neither is $P$. Since the covering conditions are satisfied here vacuously, we now construct a less trivial example, which illustrates the same thing.

EXAmPle 4.2. Let $P$ be any orthocomplete, orthomodular poset, having no atoms, which is not a lattice. (Such posets exist by the previous example.) Let $L_{2}$ be any complete lattice which is atomic and orthomodular and satisfies the covering condition. (Even a finite Boolean algebra will do.) Then clearly $P \times L_{2}$ is an orthomodular, orthocomplete poset, which is not a lattice. We claim further that it is nonatomic (but does have some atoms), satisfies the covering condition, and that $\overline{P \times L_{2}}$ also satisfies the covering condition.

Let $L_{1} \supseteq P$ be a completion for $P$. Then clearly $L_{1} \times L_{2} \supseteq P \times L_{2}$ is a completion for $P \times L_{2}$. (In fact, it follows immediately from Theorem 3.1 that if $P$ and $Q$ are partially ordered sets then $\bar{P} \times \bar{Q}$ and $\overline{P \times Q}$ are isomorphic.) Now $(x, y)$ is an atom of $L_{1} \times L_{2}$ if and only if $x=0$ and $y$ is an atom of $L_{2}$, if and only if $(x, y)$ is an atom of $P \times L_{2}$. So let $y$ be an atom of $L_{2}$ and let $(a, b) \in L_{1} \times L_{2}$ with $(0, y) \not \equiv(a, b)$. Then $y \not \equiv b$, and $L_{2}$ has the covering property, so $y \vee b>b \quad$ in $L_{2}$. Hence $(a, b)<(a, y \vee b)=(0, y) \vee(a, b) \quad$ in $L_{1} \times L_{2}$. Hence $L_{1} \times L_{2}$, and thus $\widehat{P \times L_{2}}$ satisfies the covering condition. Note that if $a \in P$ then again $(a, b)<(0, y) \vee(a, b)$ in $P \times L_{2}$, so $P \times L_{2}$ also satisfies the covering condition.

We have demonstrated in the last paragraph that $P \times L_{2}$ does have some atoms. We observe, however, that it is not atomic, for if $p \in P$ with $p \neq 0$, then $(p, 0)$ does not dominate an atom.

Example 4.3. Let $X$ be any uncountable set. Fix subsets $D_{1}, D_{2} \subseteq X$ satisfying the conditions that $D_{i} \backslash\left(D_{1} \cap D_{2}\right)$ for $i=1,2$ and $D_{1} \cap D_{2}$ are countably infinite. Let $F$ denote the set of all finite subsets of $X, C F$ denote the set of all cofinite subsets of $X, S$ denote the set of all subsets of $X$ whose symmetric difference with $D_{i}$ for $i=1$ or 2 is finite, and let $C S$ denote the set of all subsets of $X$ whose symmetric difference with $D_{i}$ for $i=1$ or 2 is cofinite. Let $P=F \cup C F \cup S \cup C S$, 
ordered by set inclusion. Define an orthocomplement ': $P \rightarrow P$ to be set theoretic complement.

It is straightforward to show that if $p, q \in P$ with $p \leqq q^{\prime}$ then $p \vee q$ exists in $P$. Hence $P$ is an orthomodular poset, and has the covering property, since $p \leqq q$ if and only if $p \cap q=\emptyset$. Furthermore, since $P$ contains all singletons, it is atomic. Now $D_{1} \cup D_{2} \notin P$, so $\left\{\{x\}: x \in D_{1} \cup D_{2}\right\}$ is a pairwise orthogonal family of elements in $P$ whose supremum fails to exist. Hence $P$ is not orthocomplete. Again, since $D_{1} \cap D_{2} \notin P$ and $D_{1}, D_{2} \in P$, it follows that $P$ is not a lattice.

We have shown that $P$ is an atomic, orthomodular poset with the covering condition, which is not orthocomplete and is not a lattice. We have left only to vertify that $\bar{P}$ satisfies the covering condition. We will do so by verifying that the power set of $X, \mathscr{P}(X)$, is a completion for $P$. To this end, let $Y \in \mathscr{P}(X)$. Then clearly $\vee\{p \in P: p \leqq Y\}=$ $\cup\{p \in P: p \subseteq Y\}=Y$. Now suppose $Y \neq \wedge\{p \in P: p \geqq Y\}$. Then there exists $a \in \cap\{p \in P: p \subseteq Y\}$ with $a \notin Y$. But $X \backslash\{a\} \in C F \subseteq P$. and $X \backslash\{a\} \supseteq Y$ and $a \notin X \backslash\{a\}$, which is a contradiction. Hence $\overline{\bar{P}}$ is isomorphic to $\mathscr{P}(X)$ and hence satisfies the covering condition.

\section{REFERENCES}

1. G. Birkhoff, Lattice Theory, (Amer. Math. Soc. Colloqu. Publ., 3rd ed. 1967).

2. P. D. Finch, On orthomodular posets, J. Austral. Math. Soc., 11 (1970), 57-62.

3. L. Haskins and S. Gudder, Heights on posets and graphs, Discrete Mathematics, 2 (1972), 357-382.

4. M. Donald MacLaren, Atomic orthocomplemented lattices, Pacific J. Math., 14 (1964), 597-612.

5. H. MacNeille, Partially order sets, Trans. Amer. Math. Soc., 42 (1937), 416-460.

Received April 16, 1974.

KÁNSAS STATE UNIVERSITY 


\section{PACIFIC JOURNAL OF MATHEMATICS}

\section{EDITORS}

RICHARD ARENS (Managing Editor)

University of California

Los Angeles, California 90024

\section{J. DugundJI}

Department of Mathematics University of Southern California Los Angeles, California 90007

D. Gilbarg and J. Milgram

Stanford University

Stanford, California 94305

\section{ASSOCIATE EDITORS}
E. F. BECKENBACH
B. H. NeumanN
F. WoLF
K. YoshiDA

\section{SUPPORTING INSTITUTIONS}

\author{
UNIVERSITY OF BRITISH COLUMBIA \\ CALIFORNIA INSTITUTE OF TECHNOLOGY \\ UNIVERSITY OF CALIFORNIA \\ MONTANA STATE UNIVERSITY \\ UNIVERSITY OF NEVADA \\ NEW MEXICO STATE UNIVERSITY \\ OREGON STATE UNIVERSITY \\ UNIVERSITY OF OREGON \\ OSAKA UNIVERSITY
}

\author{
UNIVERSITY OF SOUTHERN CALIFORNIA \\ STANFORD UNIVERSITY \\ UNIVERSITY OF TOKYO \\ UNIVERSITY OF UTAH \\ WASHINGTON STATE UNIVERSITY \\ UNIVERSITY OF WASHINGTON \\ AMERICAN MATHEMATICAL SOCIETY
}

The Supporting Institutions listed above contribute to the cost of publication of this Journal, but they are not owners or publishers and have no responsibility for its contents or policies.

Mathematical papers intended for publication in the Pacific Journal of Mathematics should be in typed form or offset-reproduced (not dittoed), double spaced with large margins. Underline Greek letters in red, German in green, and script in blue. The first paragraph or two must be capable of being used separately as a synopsis of the entire paper. Items of the bibliography should not be cited there unless absolutely necessary, in which case they must be identified by author and Journal, rather than by item number. Manuscripts, in duplicate, may be sent to any one of the four editors. Please classify according to the scheme of Math. Reviews, Index to Vol. 39. All other communications should be addressed to the managing editor, or Elaine Barth, University of California, Los Angeles, California, 90024.

100 reprints are provided free for each article, only if page charges have been substantially paid. Additional copies may be obtained at cost in multiples of 50 .

The Pacific Journal of Mathematics is issued monthly as of January 1966. Regular subscription rate: $\$ 72.00$ a year (6 Vols., 12 issues). Special rate: $\$ 36.00$ a year to individual members of supporting institutions.

Subscriptions, orders for back numbers, and changes of address should be sent to Pacific Journal of Mathematics, 103 Highland Boulevard, Berkeley, California, 94708.

PUBLISHED BY PACIFIC JOURNAL OF MATHEMATICS, A NON-PROFIT CORPORATION Printed at Jerusalem Academic Press, POB 2390, Jerusalem, Israel.

$$
\begin{gathered}
\text { Copyright (C) } 1975 \text { Pacific Journal of Mathematics } \\
\text { All Rights Reserved }
\end{gathered}
$$




\section{Pacific Journal of Mathematics}

\section{Vol. 58, No. $2 \quad$ April, 1975}

Zvi Artstein and John Allen Burns, Integration of compact set-valued functions . . . . . . . . . 297

Mark Benard, Characters and Schur indices of the unitary reflection group $[321]^{3} \ldots \ldots \ldots . .309$

Simeon M. Berman, A new characterization of characteristic functions of absolutely continuous

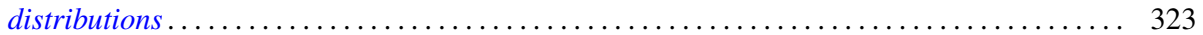

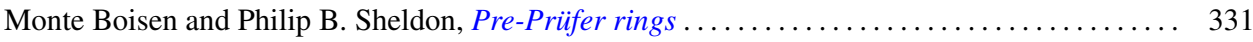

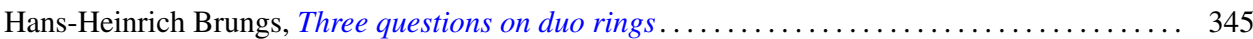

Iracema M. Bund, Birnbaum-Orlicz spaces of functions on groups................. 351

John D. Elwin and Donald R. Short, Branched immersions between 2-manifolds of higher

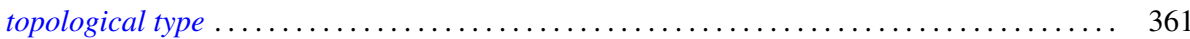

Eric Friedlander, Extension functions for rank 2, torsion free abelian groups . .......... 371

Jon Froemke and Robert Willis Quackenbush, The spectrum of an equational class of

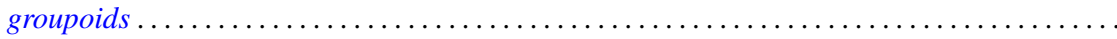

Barry J. Gardner, Radicals of supplementary semilattice sums of associative rings ...........

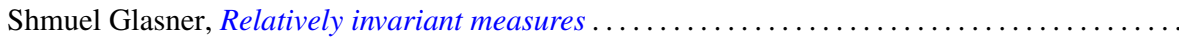

George Rudolph Gordh, Jr. and Sibe Mardesic, Characterizing local connectedness in inverse

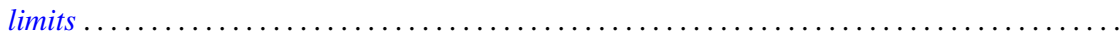

Siegfried Graf, On the existence of strong liftings in second countable topological spaces......

Stanley P. Gudder and D. Strawther, Orthogonally additive and orthogonally increasing

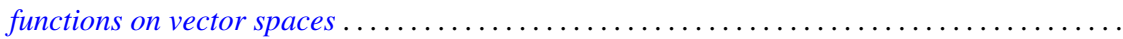

Darald Joe Hartfiel and Carlton James Maxson, A characterization of the maximal monoids and

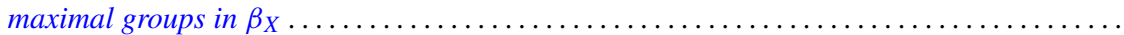

Robert E. Hartwig and S. Brent Morris, The universal flip matrix and the generalized faro-shuffle. .

William Emery Haver, Mappings between ANRs that are fine homotopy equivalences. .

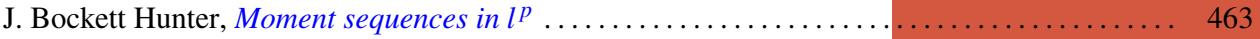

Barbara Jeffcott and William Thomas Spears, Semimodularity in the completion of a poset.... 467

Jerry Alan Johnson, A note on Banach spaces of Lipschitz functions . . . . . . . . . . . . 475

David W. Jonah and Bertram Manuel Schreiber, Transitive affine transformations on

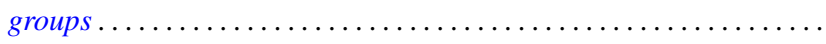

Karsten Juul, Some three-point subset properties connected with Menger's characterization of

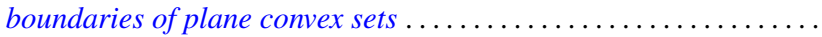

Ronald Brian Kirk, The Haar integral via non-standard analysis . . . . . . . . . . . . . 517

Justin Thomas Lloyd and William Smiley, On the group of permutations with countable

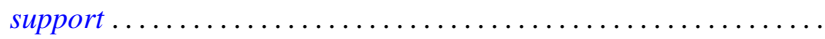

Erwin Lutwak, Dual mixed volumes .................................. 531

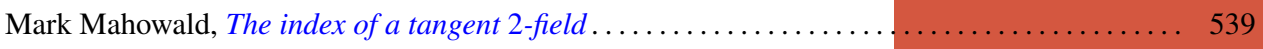

Keith Miller, Logarithmic convexity results for holomorphic semigroups . . . . . . . . . . . . 549

Paul Milnes, Extension of continuous functions on topological semigroups . . . . . . . . . . 553

Kenneth Clayton Pietz, Cauchy transforms and characteristic functions ................ 563

James Ted Rogers Jr., Whitney continua in the hyperspace $C(X) \ldots \ldots \ldots \ldots \ldots \ldots \ldots \ldots .569$

Jean-Marie G. Rolin, The inverse of a continuous additive functional . . . . . . . . . . . . 585

William Henry Ruckle, Absolutely divergent series and isomorphism of subspaces . ........ 605

Rolf Schneider, A measure of convexity for compact sets . ..................... 617

Alan Henry Schoenfeld, Continous measure-preserving maps onto Peano spaces .......... 627

V. Merriline Smith, Strongly superficial elements .......................... 643

Roger P. Ware, A note on quadratic forms over Pythagorean fields . . . . . . . . . . . . . . 651

Roger Allen Wiegand and Sylvia Wiegand, Finitely generated modules over Bezout rings . . . . 655

Martin Ziegler, A counterexample in the theory of definable automorphisms . . . . . . . . . 665 